\title{
Study of Dielectric Constant and Loss Tangent of Rochelle Salt Crystal
}

\author{
BALKRISHNA KANDPAL ${ }^{1 *}$ and TRILOK CHANDRA UPADHYAY \\ ${ }^{1}$ P.D.M. Institute of Engineering \& Technology, Karsindhu (Safidon), \\ Jind (Haryana) 126112, India \\ ${ }^{2}$ Physics Department, H.N.B. Garhwal University (A Central University), \\ Srinagar (Garhwal), Uttarakhand 246174, India \\ kandpalbk@gmail.com
}

Received 2 June 2016 / Accepted 26 June 2016

\begin{abstract}
A two sub-lattice pseudospin-lattice coupled mode model along with third and fourth order phonon anharmonic interactions terms is considered for Rochelle salt. Double Time temperature dependent Green's function method is used for development. Expression for shift, width, renormalized soft mode frequency, Curie temperature, dielectric constant and loss tangent have been derived. Model values of physical quantities are fitted. Theoretical results were compared with experimental results of literature, which show a good agreement.
\end{abstract}

Keywords: Ferroelectrics, Soft mode, Green function, Anharmonic interactions

\section{Introduction}

The Sel de seignette sodium potassium tartrate $\left(\mathrm{NaKC}_{4} \mathrm{H}_{4} \mathrm{O}_{6} \cdot 4 \mathrm{H}_{2} \mathrm{O}\right)$ or Rochelle salt is a crystal in which the ferroelectricity was discovered in1922 by Joseph Valasek. It is colorless to blue while salt crystallizing in the orthorhombic system. It is slightly soluble in alcohol but more completely soluble in water. It has been used as medicinally as a purgative but in more recent years its piezoelectric properties have been important and it has found usage in phonograph pickups and other sensing devices. Its melting point is $75^{\circ} \mathrm{C}$, boiling point $220{ }^{\circ} \mathrm{C}$. Although it is earliest ferroelectric material but is still the subject of intensive study due to its two transitions. It is ferroelectric between $255 \mathrm{~K}$ and $297 \mathrm{~K}$ showing monoclinic structure in ferroelectric phase. On deuteration the transition temperatures shift to $251 \mathrm{~K}$ and $306 \mathrm{~K}$ respectively. The theories of ferroelectric properties of Rochelle salt were initiated by Muller ${ }^{1}$. After that from microscopic point of view, Mason ${ }^{2}$ assumed that the displacement of the proton in the $\mathrm{O}_{(1)}$--- $\left(\mathrm{H}_{2} \mathrm{O}\right)_{(10)}$ hydrogen bond is the ferroelectric dipole and was able to obtain two curie points in agreement with observation. He found it is necessary, however, to assume that the dipole moment itself varies with temperature owing to the thermal expansion. Devonshire ${ }^{3}$ criticized and modified Mason ${ }^{2}$ theory, but could not reach quite satisfactory results. 
Mitsui ${ }^{4}$ proposed a two-sublattice model based on the new information obtained from $\mathrm{x}$-ray studies. Mitsui proposed that ferroelectric dipole moment arises from the ordering of protons in the $\mathrm{O}$--- $\left(\mathrm{H}_{2} \mathrm{O}\right)_{(10)}$ hydrogen bond. Later on pseudospin model was used by Blinc et al. ${ }^{5}$ to explain the two transition temperatures in Rochelle salt. Chaudhuri et al. ${ }^{6}$ have used two sublattice-pseudospin lattice coupled model along with a fourth order phonon anharmonic term. Stasyuk and Velychko ${ }^{7}$ have studied dielectric properties of Rochelle salt crystal using a four sublattice model, i.e. extension of pseudospin model. However they did not compared their results with experimental data.

Sandy and Jones ${ }^{8}$ have obtained experimental data of dielectric constant and microwave loss tangent for Rochelle salt crystal. Kamba et al. ${ }^{9}$ have made experimental studies of vibrational spectroscopy in Rochelle salt. Volkov et al. ${ }^{10}$ have made experimental study of soft mode frequency in Rochelle salt. Hlinka et al. ${ }^{11}$ have made resonant soft mode study on Rochelle salt. Shiozaki et al. ${ }^{12}$ have studied structural changes in Rochelle salt due to disordering. Noda et al. ${ }^{13}$ have studied calorimetric measurements of phase transition in ammonium mixed Rochelle say. Kikuta et al. ${ }^{14}$ have also studied mixed Rochelle salt crystals. Levitskii et al. ${ }^{15}$ have studied dielectric and piezo, electric properties of Rochelle salt crystal.

Blinc et $a l^{5}$ and Kamba et $a .^{11}$ have studied far infrared reflectivity and Raman spectroscopy in Rochelle salt. Volkov et al..$^{10}$ have made microwave dielectric measurements in Rochelle salt. Hlinka et al. ${ }^{11}$ have made inelastic neutron scattering studies in Rochelle salt crystal.

In present study, a two-sublattice pseudospin-lattice coupled mode model ${ }^{9}$ along with third-and fourth-order phonon anharmonic interactions terms ${ }^{19}$ for Rochelle salt has been used. By applying double-time thermal Green's function method ${ }^{20}$ expressions for shift, width; renormalized soft mode frequency, dielectric constant and loss tangent (microwave absorption) have been evaluated. By using model values of various physical quantities, values of soft mode frequency, dielectric constant and microwave absorption for different temperatures have been calculated and compared with experimental results of Sandy and Jones ${ }^{8}$.

Theory

Following the earlier works ${ }^{4,6}$ the two sublattice pseudospin model with symmetric doublewell potential can be expressed as

$$
H_{S}=-2 \Omega \sum_{i}\left(S_{1 i}^{x}+S_{2 i}^{x}\right)-\Delta \sum_{i}\left(S_{1 i}^{z}+S_{2 i}^{z}\right)-\sum_{i j} J_{i j}\left(S_{1 i}^{z} S_{2 j}^{z}+S_{1 i}^{z}+S_{2 j}^{z}\right)+\sum_{i j} K_{i j}\left(S_{1 i}^{z} S_{2 i}^{z}\right)
$$

Where $S_{i}^{\alpha}$ is the $\alpha$ th component of the pseudospin $\alpha=\mathrm{x}, \mathrm{y}, \mathrm{z}$ and $1=1$ or 2 corresponding to the different sublattice. The first term of $H_{S}$ is the tunnelling frequency term with tunnelling frequency $\Omega$ and the second term arises due to asymmetry of the crystal filed.

To consider the proton-lattice interaction and third and fourth order phonon anharmonic interactions we have to write the total Hamiltonian as

$$
\begin{aligned}
H & =H_{s}+H_{s-p}+H_{a n h} \\
\text { where } H_{s-p} & =-\sum_{i k} V_{i k}\left(S_{1 i}^{z} A_{k}+S_{i}^{z} A_{k}^{+}\right)
\end{aligned}
$$

and

$$
H_{\text {anh }}=\sum_{k_{1} k_{2} k_{3}} V^{3}\left(k_{1}, k_{2}, k_{3}\right) A_{k_{1}} A_{k_{2}} A_{k_{3}}+\sum_{k_{1} k_{2} k_{3} k_{4}} V^{4}\left(k_{1}, k_{2}, k_{3}, k_{4}\right) A_{k_{1}} A_{k_{2}} A_{k_{3}} A_{k_{4}}
$$


Following Zubarev ${ }^{16}$, we consider the evaluation of Green's function

$$
\begin{aligned}
& G_{i j}\left(t-t^{\prime}\right)=\left\langle\left\langle S_{i 1}^{z}(t) ; S_{j 1}^{z}\left(t^{\prime}\right)\right\rangle\right\rangle \\
& =-i \theta\left(t-t^{\prime}\right)\left\langle\left[S_{i 1}^{z}(t) ; S_{j 1}^{z}\left(t^{\prime}\right)\right]\right\rangle
\end{aligned}
$$

Differentiating Eq. (2) twice with respect to time $t$ and $t^{\prime}$ with the help of Hamiltonian (1b), Fourier transforming it and setting into Dyson's equation form ${ }^{19}$.

$$
G_{i j}(\omega)=G_{i j}^{0}(\omega)+G_{i j}^{0}(\omega) \tilde{P}(\omega) G_{i j}^{0}(\omega),
$$

We obtain Green's function

$$
G_{i j}(\omega)=-\frac{\Omega\left\langle S_{1 i}^{x}\right\rangle \delta_{i j}}{\pi\left[\omega^{2}-\widetilde{\Omega}-2 \Omega i \Gamma(\omega)\right]}
$$

where

$$
\hat{\Omega}^{2}=\widetilde{\Omega}^{2}+2 \Omega \Delta(\omega)
$$

Eq. (4) is solved self consistently, i.e. putting value of $\Delta(\omega)$ into Eq. (4) and replacing $\omega \rightarrow \widetilde{\Omega}$ are gets

$$
\begin{aligned}
\hat{\Omega}_{ \pm}^{2}=\frac{1}{2}\left[\left(\tilde{\tilde{\omega}}_{k}^{2}+\tilde{\Omega}^{2}\right) \pm \sqrt{\left\{\left(\tilde{\tilde{\omega}}_{k}^{2}-\tilde{\Omega}^{2}\right)^{2}+8 V_{i k}^{2}\left\langle S_{1}^{x}\right\rangle \Omega \omega\right\}}\right. \\
\tilde{\Omega}^{2}=\left(a^{2}+b^{2}-b c\right) \\
a=J_{0}\left\langle S_{1}^{z}\right\rangle+K_{0}\left\langle S_{2}^{z}\right\rangle+\Delta \\
b=2 \Omega \\
c=2 J_{0}\left\langle S_{1}^{x}\right\rangle+K_{0}\left\langle S_{2}^{x}\right\rangle \\
\widetilde{\widetilde{\Omega}}^{2}=\widetilde{\Omega}^{2}+2 \Omega \Delta_{s}(\omega)
\end{aligned}
$$

Dielectric constant $\in$ of Rochelle salt as (in ferroelectrics $(\in\rangle\rangle 1$ )

The tangent loss as

$$
\in(\omega)=\frac{-8 \pi N \mu^{2} \Omega\left\langle S_{1 i}^{x}\right\rangle \delta_{i j}}{\left[\left(\omega^{2}-\hat{\Omega}^{2}\right)-2 \Omega i \Gamma(\omega)\right]}
$$

$$
\tan \delta=\frac{-2 \Omega \Gamma(\omega)}{\left(\omega^{2}-\hat{\Omega}_{-}^{2}\right)}
$$

At microwave frequency $\omega\left(\omega<\left\langle\hat{\Omega}_{-}\right)\right.$

$$
\tan \delta=\frac{-2 \Omega \Gamma(\omega)}{\hat{\Omega}_{-}^{2}}
$$




\section{Results and Discussion}

In order to find temperature dependence of soft mode frequency, dielectric constant and dielectric loss tangent, the model values of physical quantities have been taken from litrature ${ }^{6}$. $T_{C 1}=255.2 \mathrm{~K} \quad T_{C 2}=296.9 \mathrm{~K}, \quad C_{1}=1830 \mathrm{~K}, C_{1}=2248 \mathrm{~K}, C_{2}=1830 \mathrm{~K}, \quad \eta=5.5 \mathrm{~cm}^{-1}$, $\Delta=0.678 \mathrm{~cm}^{-1}, \quad \Omega^{2}\left(J^{\prime}+K^{\prime}\right)^{*}=2738 \mathrm{~cm}^{-3}, \quad \Omega^{2}\left(J^{\prime}+K^{\prime}\right)=2340 K, \quad \omega_{k}=5.20 \mathrm{~cm}^{-1}$, $\Omega V_{i k}^{2}=20.9 \mathrm{~K}, A_{k} K_{B} \times 10^{17}=5.73 \mathrm{erg} / k, N=3.8 \times 10^{21} \mathrm{~cm}^{-3}, \mu=1.51 \times 10^{18} \mathrm{esu}$.

By using these values, $\left\langle S_{1}^{z}\right\rangle=\frac{J\left\langle S_{1}^{z}\right\rangle}{\widetilde{\Omega}} \tanh \frac{\tilde{\Omega}}{2 k_{B} T},\left\langle S_{1}^{x}\right\rangle=\frac{\Omega}{\widetilde{\Omega}} \tanh \frac{\tilde{\Omega}}{2 k_{B} T}$ have been calculated for different temperature T $(240-330 \mathrm{~K})$ for Rochelle salt crystal. Putting these values in Eq. (7), $\widetilde{\Omega}$ is calculated for different temperatures (240-330 K). Now values of $\hat{\Omega}_{ \pm}^{2}$, using Eq. (6), values of $\in$ using Eq. (12) and $\tan \delta$ using Eq. (14) have been calculated. Calculated values are shown in Figures 1, 2 and 3 along with data of earlier works $^{6}$ and experimental results of Sandy and Jones ${ }^{8}$.

We have derived expressions for the soft mode frequency, dielectric constant and loss tangent for Rochelle salt crystal. Double time thermal Green's function method and extended two sublattice-pseudospin-lattice coupled mode model along with third-andfourth-order phonon anharmonic interactions terms have been used in the development.

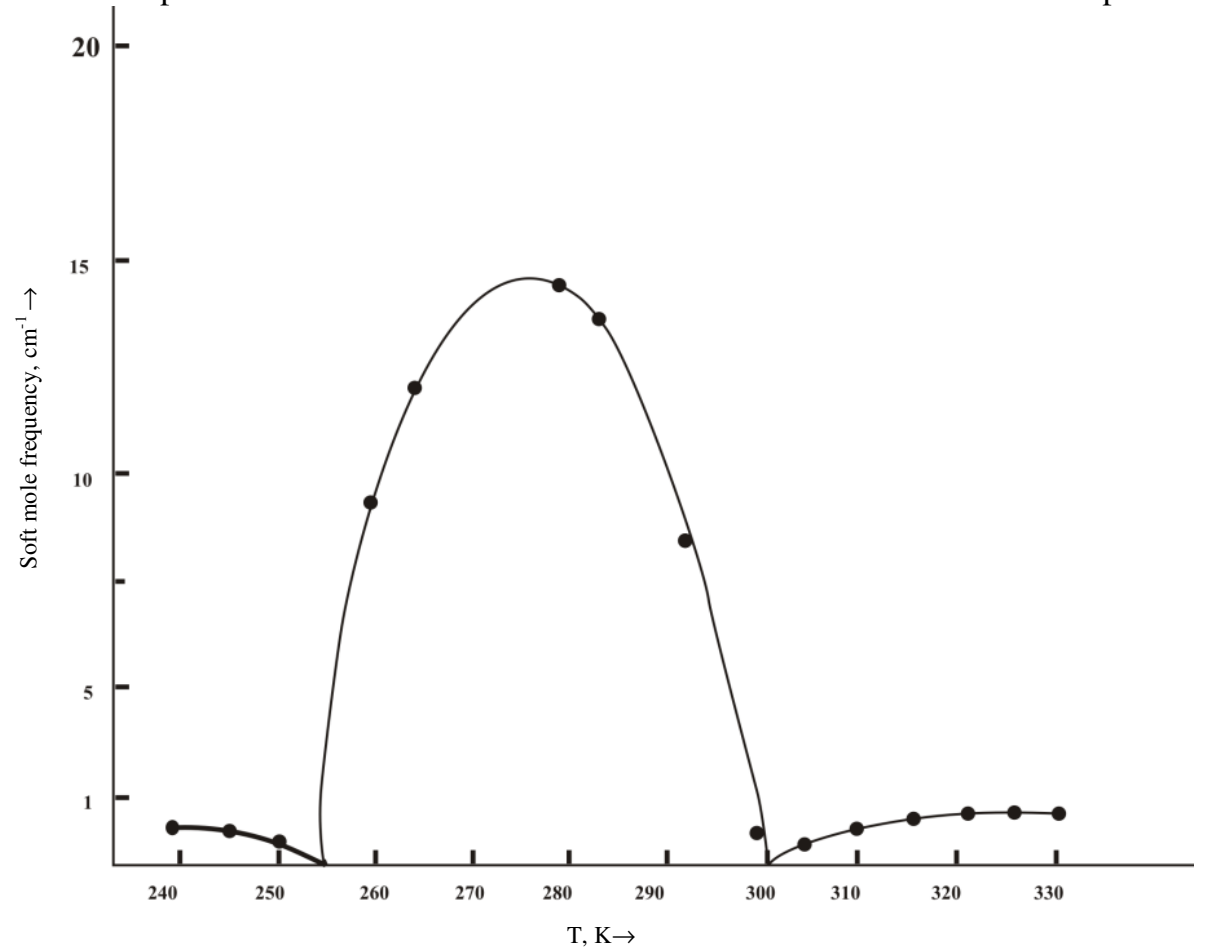

Figure 1. Temperature dependence of soft mode frequency $\hat{\Omega}\left(\mathrm{cm}^{-1}\right)$ in RS (—our results; • Experimental results of Sandy F and Jones R.V ${ }^{8}$ ) 


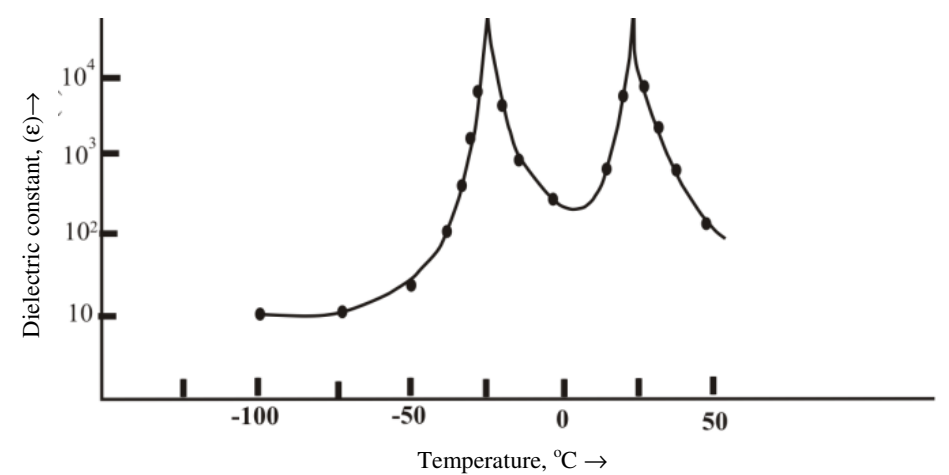

Figure 2. Temperature depenence of dielectric constant $(\epsilon)$ in RS (- our results; • Literature value $^{8}$ )

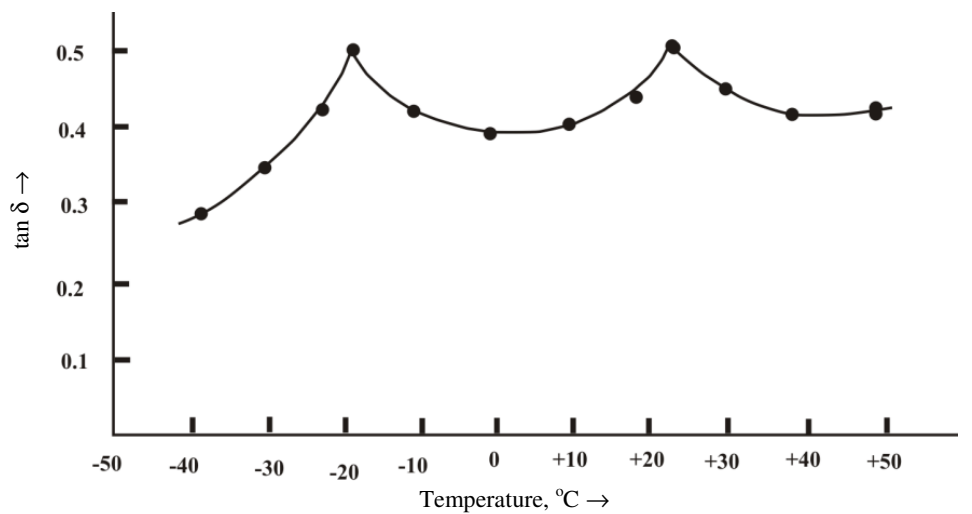

Figure 3. Temperature depenence of loss tangent $(\delta)$ in RS (— our results; $\bullet$ Literature value ${ }^{8}$ )

Model values from literature are fitted in the expressions and temperature dependence of soft mode, dielectric constant and loss tangent are calculated. Theoretical results are compared with theoretical results of earlier workers ${ }^{6}$ and experimental data of Sandy and Jones $^{8}$. Our procedure differs earlier works in the sense that they have not considered thirdorder phonon anharmonic interactions. They decoupled the correlations in the early beginning. The width and shift and the third-order phonon interactions are contributions of present work. If the width, shift and third order phonon anharmonic interactions terms are neglected from present calculations, our results reduce to the results of Chaudhari et al $l^{6}$. Our expressions predicts that loss first increases from below, upto transition then decreases and again increases upto second transition and then decreases. A transverse radiation field derives the low laying transverse mode of the material in a forced vibration. Energy in transperred from the electromagnetic field to this lattice mode and is then degraded into other vibrational modes of the material. Due to anharmonic phonon interactions, decay processes take place. For example, third-order interaction leads to the decay of a virtual phonon into two real phonons or the virtual phonon may destroyed by scattering a thermally excited phonon. Similar processes occur for fourth-order interactions.

\section{Conclusion}

As can be understood from the above results, the pseudospin-lattice coupled mode model along with third-and fourth- order phonon anharmonic interactions terms explains the dielectric 
properties of Rochelle salt clearly. The phonon anharmonic interaction terms significantly affect the temperature dependence of soft mode frequency, dielectric constant and loss tangent in Rochelle salt. These results are better than results of earlier authors, quantitatively.

\section{Acknowledgement}

The authors are thankful to Eminent Physicist Prof. B. S. Semwal, (Ex- Head, Physics Department, HNBGU Srinagar) for his valuable suggestions and Prof. U. C. Naithini (HNBGU, Pauri), Prof. R. P. Gairola (HNBGU, Srinagar), Prof. S. C. Bhatt, (Head, Physics Department, HNBGU Srinagar), Prof. K. N. Pathak (Ex VC, P.U. Chandigarh), Dr. K. S. Bartwal (RR CAT, Indore), Dr. R. P. Pant (NPL, Delhi) for their kind encouragement.

\section{References}

1. Muller K A, Helv Phys Acta, 1986, 59, 847.

2. Mason W P, Phy Rev., 1947, 72, 854; DOI:10.1103/PhysRev.72.854

3. Devonshire A F, Phil Mag., 1957, 2(20), 1027-1039;

DOI:10.1080/14786435708238209

4. $\quad$ Mitsui T, Phys Rev., 1958, 111, 1259-1267; DOI:10.1103/PhysRev.111.1259.

5. Blinc R, Petkovsek J and Zupancic I, Phys Rev., 1964, 136, A1684;

DOI:10.1103/PhysRev.136.A1684

6. Chaudhari B K, Atake T, Ganguli S and Chihara H, J Phys Soc Jpn., 1980, 49, 608; DOI: $10.1143 /$ JPSJ.49.608

7. Stasyuk I V and Velychko O V, J Phys C, 2004, 16, 1963.

8. Sandy F and Jones R V, Phys Rev., 1968, 168, 481; DOI:10.1103/PhysRev.168.481

9. Kamba S, Schaak G and Petzelt J, Phys Rev B, 1995, 51, 14998; DOI:10.1103/PhysRevB.51.14998

10. Volkov A A, Kozlov G V, Kryukova E B and Petzelt J, Zh Eksp Teor Fiz. 1986, 90(1),192-200. http://www.jetp.ac.ru/cgi-bin/dn/e_063_01_0110.pdf

11. Hlinka J, Kulda J, Kamba S and Petzelt J, Phys Rev B, 2001, 63, 052102; DOI:10.1103/PhysRevB.63.052102

12. Shiozaki Y, Shimizu K and Nozaki R, Ferroelcetrics, 2001, 261(1), 239-244; DOI:10.1080/00150190108216291

13. Noda N, Nozaki R and Shiozaki Y, Phys Rev B, 2000, 62, 12040; DOI:10.1103/PhysRevB.62.12040

14. Kikuta T, Kawabe R, Yamazaki T and Nakatami N, J Korean Phys Soc., 2003, 42, 51275 .

15. Levitskii R R, Zukek I R, Verkholyak T M and Moina A P, Phys Rev B, 2003, 67 174112; DOI:10.1103/PhysRevB.67.174112

16. Zubarev D N, Sov Phys Usp., 1960, 3, 320; DOI:10.1070/PU1960v003n03ABEH003275

17. Semwal B S and Sharma P K, Prog Theor Phys Jpn., 1974, 51(3), 639-655;

DOI:10.1143/PTP.51.639

18. Born M and Haung K, Dynamical Theory of Crystal Lattices, Oxford Press New York, 1954.

19. Upadhyay T C and Semwal B S, Pramana J Phys., 2003, 60(3), 525-533; DOI:10.1007/BF02706161

20. Upadhyay T C, Bhandari R S and Semwal B S, Pramana J Phys., 2006, 67(3), 547552; DOI:10.1007/s12043-006-0016-y

21. Kubo R, J Phys Soc Jpn., 1957, 12, 570-586; DOI:10.1143/JPSJ.12.570 